\begin{abstract}
This qualitative study was designed to look at post-partum women's body image in relation to feeding choices. Forty women aged 20-42 years, up to three years post-partum were interviewed through emails. Participants were allocated into three groups; currently breastfeeding, ex-breastfeeding, or bottle feeding. Data were analysed using thematic analysis which revealed three key themes; attitude transition, social comparisons, and media influences. Analysis suggested that women with experience of breastfeeding viewed their body more functionally which created feelings of appreciation and acceptance towards their bodies. The findings suggest that although breastfeeding does not necessarily protect women from body concerns and pressure for thinness it allows women to see their body as an 'instrument' rather than an aesthetic 'object'. Further research is required to confirm or challenge the current findings.
\end{abstract}

Key words: pregnancy; post partum body image; email interviews, thematic analysis, breastfeeding, bottle-feeding 


\section{Women's Experiences of Body Image and Baby Feeding Choices: Dealing with the}

\section{Pressure to be Slender}

Women's bodies undergo changes during pregnancy which may take them further away from the thin figure idealized in most western nations (Grogan, 2008), and some of these changes will carry over into the postpartum period. Most women adapt positively to the changes that occur during pregnancy, and some authors have suggested that pregnancy protects women from body concerns (Clark et al., 2009; Rocco et al., 2005; Skouteris et al., 2005). However, the post-partum period has been identified by many researchers as a time of augmented body dissatisfaction (Clark et al., 2009; Rallis et al., 2007; Stein and Fairburn, 1996).

One group of post-partum women who have higher levels of body satisfaction are those who are currently breastfeeding (Welsh and Hoffman, 2009), and some authors have suggested that breast feeding may provide a defence against the negative impact of pressure for slenderness. One study which looked for a relationship between body image, feeding decisions and attachment, actually found more positive pre-pregnancy body image in those who breast fed than those who chose bottle-feeding (Huang et al., 2004). Welsh and Hoffman (2009) concluded that the role of breastfeeding should be investigated more extensively. Not only does breastfeeding appear to be advantageous to a new mother's well being but it also has many advantages for a newborn including: fewer infections, lower rates of obesity and a lower chance of developing eczema (NHS, 2012) although these advantages have been queried recently (Colen and Ramey, 2014).

In a previous study we found that post-partum women did not seem to internalise the media-imposed thin ideal as expected, and that women felt sympathy for celebrities who were under pressure to look slender shortly after childbirth (Authors, 2012). Eight of the ten 
participants were currently breastfeeding, which raises the issue of whether our results would generalise to other post-partum women. The current study set out to expand on the previous findings to understand the impact feeding choice has on women's body image. Using email interviews analysed through inductive thematic analysis, this research addressed the question of whether women who have experienced breastfeeding present more positive accounts of their bodies than women who have bottle fed.

\section{Method}

\section{Participants}

40 women aged 20-42 who had given birth within the last three years were interviewed. Toby Carvery’s (2009) 'healthy eating campaign' surveyed 3,000 mums and found that two thirds of mums regained their pre-pregnancy figure within ten months; however five per cent took three years to do so. Therefore women who had given birth up to three years before the interview were considered a better and 'fuller' representation of mothers in the current population. No other exclusion criteria were applied. Participants were from similar socio-

cultural backgrounds; the only differences being that 14 were currently breastfeeding, 14 had now stopped breastfeeding and 12 were bottle feeding their child. Initially a total of 20 women were sought to aim for data saturation (Cresswell, 1998, 2002) however due to the level of interest in the research further women were interviewed.

\section{Materials/Apparatus}

A series of 16 open-ended questions were used in order to answer the research question. Examples included: "Have you seen a change in your attitude towards your body over pregnancy or the postpartum time period?", "Do you compare your body to others around you?" and "How do you feel when you look at recent celebrities who have given birth?" An 
information sheet was designed to inform women about the purposes of the study and to gain informed consent.

\section{Procedure}

Ethical approval was granted by the University ethics committee. The women were recruited using snowball sampling via poster adverts posted on several social networking pages. If someone expressed interest in the study they were asked to contact the researcher via contacts details on the poster. The participants were then sent information regarding the research, and a consent text, and were asked to reply if they had any further questions or were happy to participate. The questions were then sent in sets of three. If responses were short, participants were asked to expand on their experiences. The process was continued until all 16 questions had been asked. The participants were then thanked, the debriefing information was sent and they were reminded that they could contact the researcher at any time if they had any further questions or worries. Anonymity of data was ensured, and data were password protected. Participant's full transcripts were then emailed back to them where they were given a week for any changes (i.e. deletions) to be made.

\section{Data Analysis}

The interviews were transcribed verbatim. All data were analysed using thematic analysis, focusing on identifiable themes and patterns of living and/or behaviour (Constas, 1992). The first author familiarised herself with the data and then selected interesting pieces of text and coded these and combined them with data that were relevant to each code. Once the codes were combined, potential themes were identified. Themes were identified by "bringing together components or fragments of ideas or experiences, which often are meaningless when viewed alone" (Leininger, 1985: 60). The other two authors then read the transcripts and all authors discussed each theme. Once complete agreement was reached, a final thematic 
structure was produced and quotes selected to support each theme. Themes that emerged from the participant's stories were pieced together, defined, and named to form a picture of their experience. The analysis then shaped a storyline which is presented below.

\section{Results}

Three key themes were established from the thematic analysis: attitude transition, social comparisons and celebrity influences. Quotes are used below to indicate examples of accounts within each theme, and are identified by pseudonyms provided by the women themselves, details of months' post-partum and the women's ages.

\section{Attitude Transition}

Most participants' attitudes towards their bodies had changed as a result of pregnancy. Seven participants who were bottle feeding, nine currently breastfeeders and nine ex-breastfeeders made distinctions between their attitude pre-pregnancy and also between during pregnancy and post-pregnancy. (B/F refers to Breastfeeding, Ex-B/F refers to ex-breast feeders, and F/F refers to Bottle Feeders)

Cerys, 24, who bottle fed her child talked about her change in attitude towards her body and being 'angry' with herself now that she is not pregnant in comparison to 'loving' her body during her pregnancy. However Serena (an ex-breast feeder) talked about her body very differently:

'My body is more precious and important in its ability to carry and nurture a baby than how I look in terms of body image.'(Serena, 42, 31 months post-partum, EX$B / F)$

Serena, was much more positive towards her body when compared to Cerys and described her body as 'more precious' after giving birth. This idea is reflected by Kadam B: 
'Seeing it go through the changes pregnancy brings has made me appreciate it more and the amazing things it can do.' (Kadam B, 31, 21 months post-partum, $B / F)$

Kadam B, 31, who was breastfeeding, suggested that she had more appreciation for her body after seeing its abilities. Eleven participants from the currently breastfeeding and ten from the ex-breastfeeding group were more appreciative of their bodies after pregnancy than participants from the bottle feeding group and could see it more functionally. Participants who had bottle fed their child were less inclined to see their body in this way and were more negative of their body as can be seen in the quote from Cerys above. Whether their attitude had changed positively or negatively, for most participants a change in their attitude towards their body was evident.

\section{Social Comparisons}

When participants were asked if they made comparisons to celebrity mothers, seven participants from the currently breastfeeding group and eight participants from the exbreastfeeding group reported that celebrities were not appropriate comparison targets:

'I don't tend to compare myself to celebrities who have given birth as I find the image...is unrealistic to the average mother.'(Sarah, 21, 13 months post-partum, B/F)

Sarah, 21, suggested that comparing herself to celebrity mothers was 'unrealistic' as celebrities are not the same as 'average' mums. This perception was also shown by Jane Frost, 32:

'I don't really compare myself to celebrities because they are totally different to me and my life.' (Jane Frost, 32, 16 months post-partum, EX-B/F) 
This was compared to just two participants from the bottle feeding group who felt that celebrities were 'unrealistic' and not comparable. In fact, nearly half of participants from the bottle feeding group did compare themselves to celebrities:

'Beyonce, she gave birth to her daughter the day before I gave birth to mine and I look at my pictures now and look at hers and hate the fact she is looking like she did before and you wouldn't be able to tell she had a baby.'(Cerys, 24, 10.5 months postpartum, $F / F$ )

The proximity of Beyonce's due date made her a relevant comparison to Miss M, 26, who also compared herself to celebrities:

'I do, mainly thinking that perhaps my body should be back to looking the way it was before getting pregnant.' (Miss M, 26, 20 months post-partum, F/F)

Comparing herself to celebrities made Miss M, 26, believe that she should be back to her prepregnancy figure, creating negative feelings and unrealistic perceptions. For participants that did compare themselves to celebrities, emphasis was paid to the amount of time it had taken them to lose the weight in comparison to themselves. Only four currently breastfeeding participants and five ex-breastfeeding participants admitted comparing themselves to celebrities in this way.

\section{Celebrity Influences}

When asked how the women felt when comparing themselves to celebrities a number of differing responses emerged. For participants from the bottle feeding group emotions of jealousy and envy were more apparent: 
I I envy the ones who look amazing and I feel very jealous, at times it can make me feel embarrassed if I am looking at pictures with someone else.' (Miss M, 26, 20 months post-partum, $F / F)$

Miss M, 26, talked about feeling envious and jealous of celebrities who looked 'amazing' in pictures after giving birth and even felt 'embarrassed' if she was looking at pictures with others. Mummy M, 22, felt similar:

'I do feel a little dissatisfied and critical about my own body, even though I know celebs have an extra helping hand.' (Mummy M, 22, 8.5 months post-partum, F/F)

Even though she was aware of the extra support that celebrities receive after birth, Mummy M, 22, still felt critical about her own body in comparison. For five of the participants in the bottle feeding group, feelings of jealousy and envy arose when looking at celebrities who have given birth. As Miss M and Mummy M state, looking at celebrities for them causes negative emotions about their own bodies. However for three participants from the currently breastfeeding group and five from the ex-breastfeeding group a different emotion emerged:

'I actually feel sad for them and their babies.'(Cherry Granola, 25, 14 months postpartum, $B / F)$

Cherry Granola, 25, who was currently breastfeeding her child talked about feeling 'sad' for celebrities and their children due to added pressures they experience from being in the public eye. Sympathy was also felt by Jane Frost, 32:

'...maybe feel a bit of sympathy as I'm sure they know that if they didn't lose their baby weight and fast, that they would probably get scrutinized for it.' (Jane Frost, 32, 16 months post-partum, $E X-B / F)$ 
Jane Frost, 32, felt sympathy for celebrities due to the pressure that they are under to lose weight gained during pregnancy, in particular for celebrities who did not manage to do so. Similarly for Mac's Mummy, 33:

'Happy for them having been giving the joy of a child, but sad they are under so much pressure when normal people can just enjoy being a mummy.'(Mac's Mummy, 33, 12 months post-partum, $E X-B / F)$

Mac's Mummy, 33, who had breastfed her child, expressed mixed emotions when talking about celebrities, referring to the positivity of having a child but also the pressure for slenderness linked with fame. Interestingly no participants from the bottle feeding group felt this way.

\section{Discussion}

The findings suggest that women who had experience of breastfeeding viewed their bodies more functionally than women who bottle fed. They also had more appreciation for their bodies and the process that they had gone through to give them a child whereas women who had bottle fed their child were more negative about their own bodies. Although all participants implied that they had some degree of body dissatisfaction, women who had experience of breastfeeding implied that they were more appreciative of their body which appeared to allow them to accept the changes that had occurred through pregnancy. Women who had bottle fed their child had more negative attitudes towards their bodies which replicates previous findings (McCarthy, 1998). These findings add to the current debate around breastfeeding suggesting that women who breastfeed may be more likely to see their bodies as 'operational' as opposed to 'aesthetic' compared with women who have bottle fed their child, and this effect appears to persist even after breastfeeding has ceased. 
Some authors have suggested that western media present unrealistic expectations of new mothers by focusing on body perfection (Douglas and Michaels, 2005; Earle, 2003). Participants from the currently breastfeeding and ex-breastfeeding group felt that media representations of new mothers were 'unrealistic' and 'non-comparable'. More recent research has also suggested that women feel sympathy for celebrities and the pressure that they are under from the media (Fern et al., 2012) and this was supported by the current research. Participants felt sympathy for celebrities and also implied that they felt sad for them and their babies. What is of particular interest is that participants who felt sympathy for celebrities had experience of breastfeeding, whereas women who bottle fed who did not report feeling such sympathy. The majority of the women recruited by Fern, et al (2012) were also currently breastfeeding suggesting that breastfeeding provides a buffer and somewhat moderates the impact of the media on mother's views of their own body.

For the majority of women who experienced breastfeeding, acceptance, appreciation and a functional view of the body was found. A number of explanations could be provided for this. According to Wood-Barcalow et al. (2010), body appreciation includes four features of positive body image such as: being attentive to the body's needs, being favourable towards the body in spite of perceived imperfections, protection of the body by the dismissal of unrealistic media ideals and taking care of the body by engaging in healthy behaviours. Body appreciation is thought to predict numerous indicators of psychological well-being (e.g. life satisfaction, self-esteem, proactive coping and optimism) outside instruments that measure body dissatisfaction, implying that body appreciation is more intricate than small amounts of negative body image (Avalos et al., 2005). Women who had experience of breastfeeding were more appreciative of their bodies which may have influenced their body perceptions. Another explanation is that breastfeeding women may be viewing the body more functionally. In a study by Rubin (2006) pregnant women put their baby's and their own health above 
aesthetics. Body satisfaction during pregnancy was thought to come from a perception of increased body functionality. A positive view of the body has also been linked with physical competence (Greenleaf et al., 2009) and greater body appreciation has been linked with a focus on body function (Avalos and Tylka, 2006). Additionally, research has shown that the functional body is evaluated more favourably than the aesthetic body (Franzoi, 1995), with fewer negative evaluations made when the body is viewed more functionally (Gusella et al., 2004). This may explain why women who have experience of breastfeeding are less concerned about the appearance of their body compared to women who bottle fed their children, who appeared to view their bodies more aesthetically (and negatively).

\section{Methodological/Interpretive Issues}

As the study used email interviewing, responses from participants were relatively short which restricted the level of information obtained by the researcher. In future, researchers who conduct interviews via email might consider sending questions individually to allow participants' more time to spend on each question which may increase the level of information gained.

\section{Future Research}

It would be beneficial to conduct the current study with a larger sample of women, possibly incorporating a quantitative measure such as the Embodied Image Scale (EIS, Abbott and Barber, 2010) to gain more objective measures of body function related to body image e.g. aesthetics vs functionality. Comparing women who have experience of breastfeeding and bottle-feeding on this kind of measure would determine the generalisability of the current findings.

\section{Recommendations}


Functional dimensions of the body may be of particular significance to women who have given birth due to bodily changes that arise during pregnancy and postpartum. If viewing the body more functionally encourages women to appreciate their bodies, then pre-natal workshops for expectant mothers that encourage focus on the importance of health and wellbeing and the function of the body over and above aesthetics may be beneficial in improving their body image post pregnancy. Also, media literacy training, where women are taught how to critique media imagery around bodies, especially in relation to pressure to look slender soon after pregnancy, are likely to be helpful in promoting positive body image and wellbeing post-partum.

\section{References}

Abbott BD, Barber BL (2010) Embodied image: Gender differences in functional and aesthetic body image among Australian adolescents. Body Image, 7, 22-31

Avalos LC, Tylka TL (2006) Exploring a model of intuitive eating with college women. Journal of Counseling Psychology, 53, 486-497

Clark A, Skouteris H, Wertheim E, Paxton SJ, Milgrom J (2009) The relationship between depression and body dissatisfaction across pregnancy and the postpartum: A prospective study. Journal of Health Psychology, 14, 27-3

Colen CG, Ramey DM (2014) Is Breast Truly Best? Estimating the Effects of Breastfeeding on Long-term Child Health and Wellbeing in the United States Using Sibling Comparisons. Social Science \& Medicine. Published online January 292014

Constas M (1992) Qualitative analysis as a public event: the documentation of category development procedures. American Education Research Journal, 29, 253-266

Creswell JW (1998) Qualitative inquiry and research design: Choosing among five traditions. Thousand Oaks, CA: Sage

Creswell JW (2002) Educational research: Planning, conducting, and evaluating quantitative and qualitative approaches to research. Upper Saddle River, NJ: Merrill/Pearson Education

Douglas SJ, Michaels MW (2005) The mommy myth: The idealization of motherhood and how it has undermined women. New York: Free Press 
Earle S (2003) "Bumps and boobs": Fatness and women's experiences of pregnancy. Women's Studies International Forum, 26, 245-252.

Fredrickson BL, Roberts T (1997) Objectification theory: Toward understanding women's lived experiences and mental health risks. Psychology of Women Quarterly, 21, 173206

Fern VA, Buckley B, Grogan S (2012) Women's experiences of body image and weight loss after childbirth. British Journal of Midwifery, 20, 860-865

Franzoi SL (1995) The body-as-object versus the body-as-process: Gender differences and gender considerations. Sex Roles, 33, 417-437

Greenleaf C, Boyer EM, Petrie TA (2009) High school sport participation and subsequent psychological well-being and physical activity: The mediating influence of body image, physical competence, and instrumentality. Sex Roles, 61, 714-726

Grogan S (2008) Body image: Understanding body dissatisfaction in men, women and children (2nd ed): New York, NY, US: Routledge/Taylor \& Francis Group

Gusella J, Clark S, van Roosmalen E (2004) Body image self-evaluation colouring lens: Comparing the ornamental and instrumental views of adolescent girls with eating disorders. European Eating Disorders Review, 12, 223-229

Huang H, Wang S, Chen C (2004) Body image, maternal-fetal attachment, and choice of infant feeding method: A study in Taiwan. Birth, 31, 183-88

Leininger MM (1985) Ethnography and ethnonursing: Models and modes of qualitative data analysis. In: M M Leininger eds. Qualitative research methods in nursing Orlando, FL: Grune \& Stratton: 33-72

McCarthy ME (1998) Longitudinal study of body image and adjustment during pregnancy and the puerperium. Dissertation Abstracts International, 60, 836

NHS (2012) Why breastfeed? www.nhs.uk/Conditions/pregnancy-andbaby/pages/why-breastfeed.aspx\#close (accessed 4 April 2014)

Rallis S, Skouteris H, Wertheim EH, Paxton SJ (2007) Predictors of body image during the first year postpartum. Women \& Health, 45, 87-104

Rocco PL, Orbitello B, Perini L, Pera V, Ciano RP, Balestrieri M (2005) Effects of pregnancy on eating attitudes and disorders: A prospective study. Journal of Psychosomatic Research, 59, 175-179

Rubin L (2006) Eating for two: Body image among first-time pregnant women. Dissertation Abstracts International: Section B: Sciences and Engineering, 66 
Skouteris H, Carr R, Wertheim EH, Paxton SJ, Duncombe D (2005) A prospective study of factors that lead to body image dissatisfaction during pregnancy. Body Image, 2 , $347-361$

Stein A, Fairburn C G (1996) Eating habits and attitudes in the postpartum period. Psychosomatic Medicine, 58, 321-325

Toby Carvery (2009) Baby weight: New mums take ten months to return to their prepregnancy figure. www.dailymail.co.uk/femail/article-1162899/Baby-weight-New-mumsmonths-return-pre-pregnancy-figure.html (accessed 16 October 2012)

Welsh A C, Hoffman M A (2009) A Bio-Psychosocial Model of Body Image in New Mothers. http://drum.lib.umd.edu/bitstream/1903/9445/1/Welsh_umd_0117E_10178.pdf (accessed 8 September 2011)

Wood-Barcalow NL, Tylka TL, Augustus-Horvath CL (2010) "But I like my body": Positive body image characteristics and a holistic model for young adult women. Body Image, 7, 106- 116 